\title{
Discussion on Adolescent Internet Addiction Counseling Strategies through DEMATEL
}

\author{
Chun-An Chen \\ Department of International Business, Chung Hua University, Hsin Chu, Taiwan \\ Email: cachen@chu.edu.tw \\ Hsien-Li Lee \\ Department of Accounting, Chung Yuan Christian University, Chung Li, Taiwan \\ Email: lslej0911@yahoo.com.tw \\ Wen-Bin Yuan \\ Department of Business admin istration, Chung Hua University, Hsin Chu, Taiwan \\ Email:m09819022@chu.edu.tw
}

\begin{abstract}
Internet addiction and other such problems stem from the high frequency of Internet usage of adolescents. The study aims at working out the counseling strategy proposed to prevent internet addiction among the youth. In this study, DEMATEL was the avenue for creating a discussion with families, schools, the society and analyzing regulations \& policies so as to find out the cause-and-effect relationship among them and work out the most efficient counseling strategy. The study result shows that the family plays the most crucial role in curing the adolescents' Internet addiction through action.
\end{abstract}

Index Terms-Internet Addiction, DEMATEL, Adolescent Counseling.

\section{INTRODUCTION}

\section{A. Influence of Internet}

In the 21st century, the Internet has made a huge impact in every aspect of people's lives and internet use is steadily increasing. The sample survey of Taiwan Network Information Center [1] indicates that there are about 16,220,000 Internet users in Taiwan as of February 2010, about 400,000 more than the $15,820,000$ of 2009. Those who are over 12 and have once been Internet users account for $72.56 \%$ of the total population in Taiwan. From the perspective of the interviewees' ages, the rate of internet use among the four intervals which consists of age 12 14, age 15 19, age $20 \sim 24$ and age $25 \sim 34$, are all above $94.54 \%$. The above mentioned data shows that the Internet is commonly used by Taiwanese and is more popular with the population aged 12 to 34. Due to the continuous increase of Internet use and the characteristics of the Internet, namely anonymity, virtuality, convenience, trip, etc. [2], a lot of similar problems are brought about subsequently. For example, Joinson [3] pointed out the misconducts such as dirty words, abuses and obloquy, as well as the anonymity offered by the network caused the effect of de- individualization. Young [4] pointed out that Internet addiction was like the symptom of impulse-control disorders, in other words, Internet addiction would result to the involuntary Internet use of an individual, making him unable to extricate himself or herself from the Internet; anyhow, everyone, regardless of his status and role, should know when to stop in case of any behavior of addiction that has tremendous influence on one's daily life, so as to avoid physical and psychological harms. This has also become a topic worth studying and discussing in our society.

\section{B. Study Purpose}

Although researches and discussions on adolescent Internet addiction are increasing in Taiwan, most of them are qualitative discussions on the said phenomenon from the perspective of medical pathology. This study will be based on the previous studies. Due to the complicated relationship among the factors that contribute to adolescent Internet addiction and the relevance of each dimension and criterion, complex problems can be analyzed through DAMATEL in the study to quantify the degrees of correlativity and influence of all elements and understand the characteristics of its cause-and-effect relationship. For instance, by using DEMATEL, Shieh, Wu and Huang [5] discussed the issues related to medical service quality, Tseng \& Lin [6] studied the cause-and-effect relationship of the management issues and Fekri, Aliahmadi and Fathian [7] found out the cause of the incident and improved the decision quality. Therefore, it is appropriate to discuss the is sue concerning adolescent Internet addiction through DEMATEL; moreover, the data obtained from discussing the issues related to adolescent Internet addiction from the perspectives of the family, the school, the society, the environment, etc. is expected to assist social counselors and parents as well as teachers at school in positively contributing towards Internet addiction counseling.

\section{Study Organization}

First, we study a lot of literature, including the 
researches about the internet addiction and counseling strategies. Second, we create the study framework and develop the DEMATEL expert questionnaire. Then we collect the opinions of experts. The data were analyzed using the "Super DEMATEL v1.6" program [8]. Finally, according to the results of analyzing, we present the conclusions and suggestions.

\section{LITERATURE DISCUSSION}

\section{A. Meaning of Internet Addiction}

With the increase in the convenience offered by the internet, decrease of the online-service expenses and the popularization of computers, Internet addiction is getting more and more serious in the society. All internet activities including information collection, receiving and sending emails, network community, real-time communication software, online games, etc. can bring great fun but also result in many users' excessive use and even dependence on the network, which consequently causes Internet addiction, not only influencing the daily life but also likely to harm individual physical and mental health. Young [9] pointed out eight criteria for judging Internet addiction. He suggests that Internet addiction could be determined as long as five or more questions are answered "yes" in the following eight statements: (1) Do you think you are immersed in the Internet (including recalling the previous online activities or expecting the next surfing)? (2) Do you think you are not satisfied unless you have more time to surf the Internet? (3) Have you once failed in attempting to control, pause or stop online activities? (4) Do you feel uncontrollable, sad, depressed or illtempered when you try to pause or stop online activities? (5) Do you spend much more time than you scheduled on the Internet? (6) Are you willing to take the risk of losing important interpersonal relationship, jobs, learning or working opportunities caused by surfing the Internet? (7) Have you lied to your family, friends, tutors or others who are concerned about you to conceal the fact that you are surfing the Internet? (8) Have you ever escaped from troubles or relieved discomfort by surfing?

Internet addiction can be divided into five types according to the use content [10] : (1) sexual addiction to the Internet: attracted by contents related to sex on the network or addicted to the erotic activities found online. (2) Relationship addiction to the Internet: addicted to interpersonal relationship activities online. (3) Information overload: addicted to online shopping \& transactions, gambling, online games and other such activities. (4) Information overload from the Internet: addicted to online information searching or collection and so on. (5) Computer addiction online: addicted to computer operation and application related to network. The person concerned may have two or more addictions among these five aforesaid types.

For people who use the Internet, some would be addicted but some could extricate himself from it.
Therefore, Grohol [11] put forward such an explanation model, namely, that network users would experience three phases when they were engaged in online activities:

(1) Enchantment: Spend a lot of time to explore and adapt to new online activities;

(2) Disillusionment: The network use is gradually decreased with the familiarity with and adaptation to the online activities;

(3) Balance: Able to appropriately distribute time on online activities. He/she deems that most users can progress to the third phase, while some users will get stuck in phase I and need others' help to move on.

\section{B. Internet Addiction Counseling Strategy}

The study of Wei, Li and Wang [12] indicates that adolescent Internet addiction is influenced by such environmental factors such as family interaction, school adjustment, etc. which must be taken into consideration while planning the counseling strategy and treatment. Parents and teachers should positively participate in and cooperate with the planning, meanwhile, the environment itself needs to be adjusted to improve the adolescent's positive feelings and acknowledgement of the Internet. Furthermore, healthy entertainment can be cultivated by combining the relevant agency resources and through interesting after-class activities, so as to reduce their dependence on the Internet. Zastrow, Kirst and Karen [13] put forward that the human activity and the social environment are, so to speak, interrelated and interdependent. The human behavior, which is gradually formed from the culture, language, custom, etiquette concept, etc. through the baptism of time, is one of the most essential elements for constituting the social environment. What is society literally? Society is an environment where many people live in, which includes human life and all of their activities. While the human activity must give into the restriction of the environment as well, the cultures and customs in various regions are different accordingly; with continuous interaction between humans and environment, all activities conducive to human life are created, which also give rise to the local social culture. From all of the things aforementioned, we can come up with the conclusion as stated in the first sentence that the human activity and the social environment are, so to speak, interrelated and interdependent. In addition, the adolescent's use of the Internet may be restrained effectively if relevant policies and laws are formulated by the government.

Consequently, concerning the counseling strategy for Internet addiction, four dimensions are sorted out from the previous documents in this study, namely the school, the family, the society and policies and laws in order. The study of Wang [14] suggests that the counseling strategies regarding the circumstances in the family and the school include (1) strengthening the establishment of the diverse relationship and inculcating the skills necessary to maintain relationship and communication: the assistance provided to the adolescents in cultivating diverse interpersonal relationship and learning the skill 
of interpersonal communication can not only prevent their excessive dependence on the Internet but also teach them to deal with setbacks or damages caused by online or actual interpersonal interaction by learning the ways on how to maintain relationships and manage conflicts; (2) clarifying the adolescent life goals and learning time management: the reason for the adolescent's excessive use of the Internet may be due to the fact that they have too much idle time or they want to relieve pressure by surfing the Internet, therefore, time management and handling pressure well play a role in offering assistance to the adolescent; (3) proper specification and restraint by others: for example, the adolescent's family and elders may adjust the placement of the computer which is more appropriate to be situated at a public place, thus to increase the opportunities for the family members to take part in the activity or interact together and monitor the adolescent to see whether his/her use of the Internet is proper; (4) learning effective methods to communicate with the adolescent: avoid tension in the parent-child relationship caused by the parents' restraint in the children's use of the Internet. Moreover, the physical and mental development of the adolescent can be understood and the parent-child relationship can be improved through effective communication; (5) proper environmental planning: establishing a high-quality and healthy network environment and so on in the family and the school; (6) providing selective and substitute activities including entertainment, activities for improving interpersonal relationships, activities for improving the sense of self-esteem and responsibility, etc.; (7) offering and strengthening the adolescent's selfcontrol ability, perceptiveness and other abilities: generally speaking, the self-control ability and perceptivity of the adolescents are underdeveloped in school, so relevant curriculums should be planned to improve the adolescents' relevant abilities.

Additionally, in order to create a high-quality and healthy social network environment, the counseling strategies concerning the circumstances of the society and policies \& laws include (1) regulations on management and counseling of the Internet cafes: offering a relatively healthy community for the Internet environment; (2) relevant mechanis ms and regulations for strengthening investigations on cyber crime: offering a relatively safe network activity space; (3) creating a high-quality and healthy network environment: building high-quality and healthy websites; (4) improving highquality network culture: advocating the concept of properly using the Internet.

\section{STUDY METHOD}

\section{A. Study Process}

The theme of the study is to discuss the Internet addiction counseling strategies for the youth. After the study background, motive and purpose are determined, the documentary analysis method is adopted to summarize the reasons behind the Internet addiction and the counseling strategies formulated in each study so as to construct the preliminary analysis framework of the study, and then make out a formal study framework after absorbing the experts' suggestions. Since the relevance of the factors indicated in the strategies for remedying adolescent Internet addiction is complicated and each dimension and criterion are correlated, DEMATEL and Maximum Mean De-Entropy Algorithm are applied in the study. DEMATEL is mainly used to analyze complex problems, quantize the degrees of relevance and influence of all elements and understand their causeand-effect structural relationship; MMDE tries to find out the unique threshold to screen the critical cause-andeffect relationship of DEMATEL based on the concept of "entropy". Through this way, the optimu m counseling strategy can be effectively sought and the conclusion and suggestion will be proposed according to the analysis results.

\section{B. Study Framework}

In this study, the documents related to Internet addiction are summarized, including the constituting factors, counseling strategies for Internet addiction, etc. forming a preliminary counseling strategy framework; in addition, the study framework is determined through discussions with experts and integration of the suggestions given by them, mainly aiming at formulating the optimum counseling strategy. The study framework is divided into two levels: the first-level counseling strategy dimension includes four criteria, namely, the family, the school, the society and laws \& policies; the second-level counseling index consists of eleven indexes subdivided from the four dimensions of the first level. Table I shows the integral study framework.

TABLE I. STUDY FRAMEWORK

\begin{tabular}{|c|c|}
\hline & Strategy \\
\hline $\begin{array}{l}\text { A. } \\
\text { Family }\end{array}$ & $\begin{array}{l}\text { a1. Proper regulation and restraint by } \\
\text { others }\end{array}$ \\
\hline & $\begin{array}{l}\text { a2. Learning effective methods to } \\
\text { communicate with the adolescent }\end{array}$ \\
\hline & a3. Proper environment planning \\
\hline $\begin{array}{l}\text { B. } \\
\text { School }\end{array}$ & $\begin{array}{l}\text { b1. Clarifying the adolescent's life } \\
\text { goals and learning time management }\end{array}$ \\
\hline & $\begin{array}{l}\text { b2. Providing the adolescent with } \\
\text { selective and substitute activities }\end{array}$ \\
\hline & $\begin{array}{l}\text { b3. Strengthening the adolescent's } \\
\text { establishment of interpersonal } \\
\text { relationships and training of } \\
\text { communication skills }\end{array}$ \\
\hline & $\begin{array}{l}\text { b4. Strengthening the adolescent's } \\
\text { self-control ability and percep tivity }\end{array}$ \\
\hline $\begin{array}{l}\mathrm{C} . \\
\text { Environment }\end{array}$ & $\begin{array}{l}\text { c1. Constructing a high-quality and } \\
\text { healthy network environment }\end{array}$ \\
\hline & $\begin{array}{l}\text { c2. Improving high-quality network } \\
\text { culture }\end{array}$ \\
\hline $\begin{array}{l}\text { D. } \\
\text { Laws }\end{array}$ & $\begin{array}{l}\text { d1. Relevant mechanisms and } \\
\text { regulations for strengthening the } \\
\text { investigation on cyber crime }\end{array}$ \\
\hline & $\begin{array}{l}\text { d2. Regulations on management and } \\
\text { counseling of Internet cafes }\end{array}$ \\
\hline
\end{tabular}




\section{DEMATEL}

DEMATEL originated from Geneva Research Center Battelle Association [15, 16] in 1973. At that time, it was used to study complex and difficult problems in the world, aiming at assisting in gathering the world's problems and seeking the integrated solutions, thus to increase the understanding of the World problematique and encourage better knowledge exchange among all regions of the world through this method. DEMATEL can be used to effectively comprehend the complex cause-and-effect relationship structure and calculate the causality relationships of all elements by making use of the matrix and relevant mathematical theories via the influence between elements; moreover, the degree of cause-and-effect influence is expressed in figures. DEMATEL is widely applied in enterprise planning and policy decision making, urban planning and design, geographic and environmental assessment and other fields. DEMATEL is executed in these following steps: [17]

(1) Definition of Elements: List and define the elements in the system.

(2) Judgment of Relationship: Judge the relationship between elements according to the subjective recognition of experts on the problems.

The assessment degree is classified into four types: 0uninfluenced; 1- slightly influenced; 2-influenced; 3greatly influenced.

(3) Generation of Direct-relation Matrix: When the number of the criteria is " $n$ ", the criteria are compared based on their relation and degree of influence to get $\mathrm{n} \times \mathrm{n}$ matrix, called direct-relation matrix and expressed by “Z”. Zij represents the degree of influence of criteria $\mathrm{i}$ on criteria $\mathrm{j}$. [the diagonal element $\mathrm{Zij}=0$ if $\mathrm{i}=\mathrm{j}$ ]

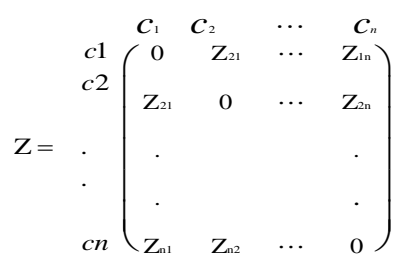

(4) Calculation of Standardized Direct-relation Matrix:

$$
\text { Let } \lambda=\frac{1}{\max _{1 \leq i \leq n}\left(_{j-1}^{n} \sum_{i j}\right)}
$$

A standardized direct-relation matrix $\mathrm{X}$ can be obtained by multiplying the elements of the whole matrix by $\lambda$ that is a quotient of 1 divided by the maximumsum of lines in matrix $Z$, namely $X * Z$.

(5) Calcu lation of Direct / Indirect Matrix:

The direct / indirect matrix $\mathrm{T}$ can be obtained from the following formula.

$$
T=\lim _{k \rightarrow \infty}\left(I+X+X^{2}+\ldots+X^{K}\right)=X(I-X)^{-1}
$$

(6) Calculation of Prominence and Relevance: Let Tij $(\mathrm{i}, \mathrm{j}=1,2, \ldots, \mathrm{n})$ be the elements in $\mathrm{T}$ and the sums of rows and lines are expressed by $\mathrm{Di}$ and Rj respectively.

$$
\begin{aligned}
& D_{i}=\sum_{i=1}^{n} \operatorname{Tij}(i=1,2, \ldots \ldots, n) \\
& R_{j}=\sum_{j=1}^{n} \operatorname{Tij}(j=1,2, \ldots ., n)
\end{aligned}
$$

$\mathrm{D}_{\mathrm{i}}$ means the sum taking the element $\mathrm{i}$ as the reason and which influences other elements. $R_{j}$ means the sum taking the element $\mathrm{j}$ as the result and which is influenced by other elements. $(D+R)$ is called prominence, referring to the overall degree of influencing and being influenced by the element. (D-R) is called relation. If (D-R) is positive, the element tends to be result category; if (D-R) is negative, the element tends to be influence category.

(7) Drawing of Causal Diagram: In the causal diagram, the horizontal axis $(D+R)$ is called prominence, representing the intensity of relationships among factors; the longitudinal axis (D-R) is called relation, representing the said element's degree of influencing and being influenced. The causal diagram may help the decision makers to take the appropriate measures in accordance with the result or influence category in the criteria.

\section{Maximum Mean De-Entropy Algorithm}

Entropy derived from the research on information theory was introduced by C. Shannon in 1948. A.N. Kolmogorov, a mathematician from Soviet Union gave the concept of the dynamical power system entropy on that basis in 1958 [18].

Viewing the relevant DEMATEL documents, the methods for setting the threshold include geometric mean, arithmetic mean, expert opinion methods, or unexplained. Li put forward MMDE in 2009, which simultaneously assesses the dominating node and influencing node, considering not only the degree of influence of the specific variables on other variables but also the degree of influence of other variables on the said variable. Meanwhile, the unique threshold can be obtained, which is beneficial for the researchers who solve problems to select a consistent threshold in case they are faced with the aforementioned problems.

The definitions of the MMDE method [19] are described below:

Definition 1: The information entropy of a discrete random variable $X$, that can take the range of possible values $\left\{\mathrm{x}_{1}, \cdots, \mathrm{x}_{\mathrm{n}}\right\}, \mathrm{Pi}$ is the probability of $\mathrm{X}_{\mathrm{i}}$, defined as $\mathrm{H}\left(\mathrm{p}_{1}, \mathrm{p}_{2}, \cdots, \mathrm{p}_{\mathrm{n}}\right)=-\sum \mathrm{p}_{\mathrm{i}} \lg \mathrm{p}_{\mathrm{i}}, \mathrm{H}\left(\mathrm{p}_{1}, \mathrm{p}_{2}, \cdots, \mathrm{p}_{\mathrm{n}}\right)$ is the level of entropy, subject to constraints $\sum_{\mathrm{i}=1}^{\mathrm{n}} \mathrm{p}_{\mathrm{i}}=1,\left(\mathrm{p}_{\mathrm{i}} \lg \mathrm{p}_{\mathrm{i}}=0\right.$, if $\left.\mathrm{p}_{\mathrm{i}}=0\right)$.

If the same distribution is assigned to the pi and entropy value is maximum, it is denoted as $\mathrm{H}$. Another measure for the decreased level of entropy- de-entropy defined as $H(1 / n, 1 / n, \ldots, 1 / n)$.

Definition 2: For a given fin ite discrete scheme of $\mathrm{X}$, the de-entropy of $\mathrm{X}$ is denoted as $\mathrm{H}^{\mathrm{D}}$ and defined as $\mathrm{H}_{\mathrm{n}}{ }^{\mathrm{D}}=\mathrm{H}(1 / \mathrm{n}, 1 / \mathrm{n}, \ldots, 1 / \mathrm{n})-\mathrm{H}\left(\mathrm{p}_{1}, \mathrm{p}_{2}, \ldots, \mathrm{p}_{\mathrm{n}}\right)$

As we mentioned above, when the probability of all elements are equal, the $\mathrm{H}$ is maximum. It is easy to determine whether the value of $\mathrm{H}^{\mathrm{D}}$ is equal to or larger 
than 0 . The $\mathrm{H}^{\mathrm{D}}$ can explain a lot of useful in formation derived from a specific dataset, which reduces the "uncertainty" of information.

Definition 3: The (i, j) element of the matrix $\mathrm{T}$ is denoted as tij and directly affects the relationship from factor xi to factor xj. For each $t_{i j}$, the factor $x i$ is defined as a dispatch-node and factor $x_{j}$ is defined as a receivenode with respect to $t_{i j}$. By Definition of $\mathrm{H}^{\mathrm{D}}$, an $n \times n$ total relation matrix $\mathrm{T}$ can be considered as a collection (set $\mathrm{T}$ ) with $\mathrm{n} 2$ pair ordered elements. Each subset of the set $\mathrm{T}$ can be divided into two sets: an ordered dispatch-node set and an ordered receive-node set. For an ordered dispatch-node set, the frequency of the different elements of the set are counted. If the finite cardinality of an order dispatch-node set is $\mathrm{m}$ and the frequency of element $x_{i}$ is $k$, the corresponding probability of $x_{i}$ as Eq is assigned $\mathrm{p}_{\mathrm{i}}=\mathrm{k} / \mathrm{m}$.

MMDE is executed as follows: [20]

[1] Convert the Overall Influence Matrix into an Ordered Set of Three Elements

The overall influence matrix $\mathrm{T}$ is reformed as an ordered set $\mathrm{T}=\{\mathrm{t} 11, \mathrm{t} 12, \ldots, \mathrm{t} 21, \mathrm{t} 22, \ldots, \mathrm{tnn}\}$, then the elements in set $\mathrm{T}$ are rearranged in descending order and converted into an ordered set $\mathrm{T}^{*}$ of three elements ( $\mathrm{tij}, \mathrm{xi}, \mathrm{xj})$. The three elements (tij, xi, xj) in the ordered set respond to the influence value, dispatch node and influenced node in the overall in fluence matrix $\mathrm{T}$.

[2] Build the Dispatch-Node Set

The second element is taken out of $\mathrm{T}^{*}$ to serve as the set TDi of the dispatch node. TDi $=\{x i\}=\{x 1, x 2, \ldots, x n\}$.

[3] Calculate the Average Entropy Difference of the Dispatch-Node Set

Take out the first $\mathrm{t}$ elements from TDi as a new set $\mathrm{TtDi}$ and find out the probability of each element. Then calculate the entropy difference $\mathrm{HtDi}$ of the set TtDi by applying Steps 1 and 2. The value of $\mathrm{t}$ starts from 1 , increased by equal difference value 1 , with the final value being $C$ (TDi). Calculate the average entropy difference through the formula MDEtDi=HtDi/N(TtDi). The repeated times should be ignored as the dispatch node appears only once in the overall relation diagram. Therefore, the divisor should be taken as the sum of the number of various elements N(TtDi) in the set instead of the sum of all nu mbers C(TtDi) in the set.

[4] Find out the Maximum Average Entropy Difference

Find out the maximum average entropy difference and the corresponding dispatch-node set TtDi which responds to the dispatch-node set of the maximum average entropy difference, namely TmaxDi.

[5] Find out the Set of the Influenced Nodes and Calculate the Maximum Average Entropy Difference
Repeat Steps 2 to 4 to find out the ordered set TRe of the influenced nodes, the maximu m average entropy difference and the set of influenced nodes corresponding to the maximum average entropy difference, namely TmaxRe.

[6] Find out the Threshold

The elements including all dispatch nodes TmaxDi and influenced nodes TmaxRe in $\mathrm{T}^{*}$ are selected as the subset TTh of which the minimum influence value is the threshold.

\section{EMPIRICAL STUDY}

In this study, the 11 criteria in the study framework diagram are summarized as four dimensions to serve as the basis of the design of the questionnaire and the questionnaire scale is divided into $\ulcorner 0\lrcorner,\ulcorner 1\lrcorner,\ulcorner 2\lrcorner$, $\ulcorner 3\lrcorner$ and $\ulcorner 4\lrcorner$, respectively representing [uninfluenced], [slightly influenced], [influenced], [greatly influenced] and [extremely influenced]. Then, the DEMATEL expert questionnaires are delivered, of which the main objects sampled are those who have long been engaged in adolescent counseling and have more than five years of seniority. According to Teng [21], 5 to 15 experts will be appropriate for group decision making. There are a total of eight questionnaires distributed in this study.

\section{A. Analysis on the Evaluation of Influence between DEMATEL Dimensions}

The direct / indirect-relation matrix of dimensions is obtained by summarizing the results of the questionnaire survey which are sorted out as shown in Table II.

TABLE II. DIRECT/INDIRECT-RELATION MATRIX DIAGRAM OF DIMENSIONS

\begin{tabular}{|l|c|c|c|c|}
\hline & Family & School & Environment & Laws \\
\hline Family & 2.3141 & $\mathbf{2 . 7 3 8 6}$ & $\mathbf{2 . 9 6 7 0}$ & $\mathbf{2 . 8 5 7 5}$ \\
\hline School & 2.3538 & 2.2744 & $\mathbf{2 . 7 4 3 1}$ & 2.6122 \\
\hline Environment & 2.3920 & 2.5305 & 2.5473 & 2.7060 \\
\hline Laws & 2.1932 & 2.3662 & 2.6292 & 2.2746 \\
\hline
\end{tabular}

Boldfaces represent values great er than the threshold (2.7386).

Calculate Table II and sort the sums of rows and lines to get Table III as below.

TABLE III. RELATION MATRIX OF OVERALL INFLUENCE OF DIMENSIONS

\begin{tabular}{|l|c|c|c|c|}
\hline & $\mathrm{D}$ & $\mathrm{R}$ & $\mathrm{D}+\mathrm{R}$ & $\mathrm{D}-\mathrm{R}$ \\
\hline Family & 10.8771 & 10.8867 & 21.0624 & 1.6241 \\
\hline School & 10.1757 & 10.4502 & 20.1301 & 0.0738 \\
\hline Environment & 9.9835 & 9.9097 & 19.9134 & -0.7108 \\
\hline Laws & 9.4631 & 9.2530 & 19.8932 & -0.9871 \\
\hline
\end{tabular}


The above Table III indicates the relationship of influence among the four dimensions; Table II shows the relevance degrees of the four dimensions : [environment], [family], [laws] and [school] arranged according to the prominence of the sums of rows and lines (D+R), while in terms of the difference of the sums of rows and lines (D-R), the relevance degrees of the four dimensions are [family], [school], [environment] and [laws]. The D+R value of the [environment dimension] is 21.0624, the maximum of the four dimensions, indicating its degree of relevance is the greatest; and the $\mathrm{D}-\mathrm{R}$ value is negative, indicating that it is likely to be affected by other dimensions. The D+R value of the dimension [family] is 20.1301, which means a great degree of relevance; D-R value is positive, the maximum among the four dimensions, showing that the family is the main dimension influencing other dimensions. The relational diagram of influence between dimensions (Fig. 1) and causal diagram of dimensions (Fig. 2) are drawn according to the above Tables II and III.

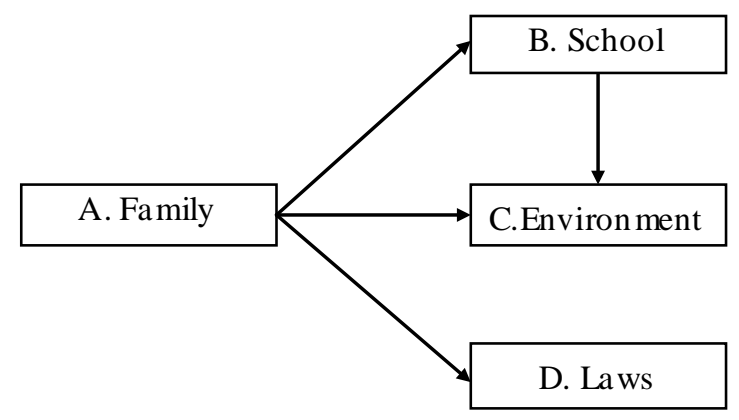

Figure 1. Relational Diagram of Influence between Dimensions

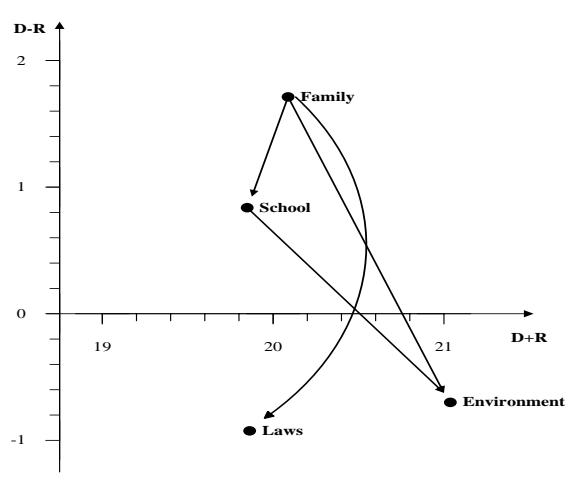

Figure 2. Causal Diagram of Dimensions

Fig. 2 shows that the dimension of [family] is the most important factor, and [school] comes second; [family] will influence [school], [environment] and [laws] at the same time; therefore it is necessary to start from the family and then the school while formulating the counseling dimension of adolescent Internet addiction.

\section{B. Analysis on Evaluation of Influence Between Criteria}

The direct / indirect-relation matrix is obtained by summarizing the results of the questionnaire survey which are sorted out as shown in Table IV.
TABLE IV. DIRECT/INDIRECT-RELATION MATRIX OF CRITERIA

\begin{tabular}{|c|c|c|c|c|c|c|c|c|c|c|c|}
\hline & a1 & a2 & a3 & b1 & b2 & b3 & b4 & c1 & c2 & d1 & d2 \\
\hline a1 & 0.29 & $\mathbf{0 . 3 1}$ & $\mathbf{0 . 3 2}$ & $\mathbf{0 . 3 0}$ & 0.28 & 0.24 & 0.28 & $\mathbf{0 . 3 0}$ & 0.24 & $\mathbf{0 . 2 9}$ & 0.27 \\
\hline a2 & $\mathbf{0 . 3 4}$ & 0.17 & 0.24 & 0.27 & 0.21 & 0.22 & 0.23 & 0.21 & 0.17 & 0.20 & 0.19 \\
\hline a3 & $\mathbf{0 . 3 4}$ & 0.24 & 0.17 & 0.23 & 0.23 & 0.19 & 0.21 & 0.26 & 0.21 & 0.23 & 0.22 \\
\hline b1 & $\mathbf{0 . 3 2}$ & 0.25 & 0.23 & 0.17 & 0.25 & 0.23 & 0.23 & 0.21 & 0.18 & 0.18 & 0.18 \\
\hline b2 & $\mathbf{0 . 3 1}$ & 0.24 & 0.23 & 0.26 & 0.15 & 0.20 & 0.21 & 0.22 & 0.19 & 0.20 & 0.19 \\
\hline b3 & 0.28 & 0.24 & 0.19 & 0.22 & 0.20 & 0.12 & 0.21 & 0.20 & 0.16 & 0.17 & 0.16 \\
\hline b4 & 0.28 & 0.21 & 0.18 & 0.22 & 0.18 & 0.18 & 0.13 & 0.19 & 0.17 & 0.17 & 0.17 \\
\hline c1 & 0.27 & 0.18 & 0.21 & 0.18 & 0.19 & 0.16 & 0.18 & 0.15 & 0.19 & 0.22 & 0.21 \\
\hline c2 & 0.25 & 0.19 & 0.21 & 0.18 & 0.17 & 0.15 & 0.17 & 0.25 & 0.12 & 0.22 & 0.22 \\
\hline d1 & 0.28 & 0.18 & 0.20 & 0.17 & 0.17 & 0.14 & 0.17 & 0.26 & 0.19 & 0.14 & 0.22 \\
\hline d2 & $\mathbf{0 . 2 9}$ & 0.18 & 0.20 & 0.17 & 0.17 & 0.15 & 0.17 & 0.26 & 0.20 & 0.24 & 0.14 \\
\hline \multicolumn{7}{|c|}{ Boldfaces represent values greaterthan the threshold (0.2875). }
\end{tabular}

TABLE V. OVERALL INFLUENCE OF CRITERIA

\begin{tabular}{|l|l|l|l|r|}
\hline & $\mathrm{D}$ & $\mathrm{R}$ & $\mathrm{D}+\mathrm{R}$ & \multicolumn{1}{c|}{$\mathrm{D}-\mathrm{R}$} \\
\hline $\mathrm{a} 1$ & 3.1087 & 3.2575 & 6.3662 & -0.1487 \\
\hline $\mathrm{a} 2$ & 2.4553 & 2.4019 & 4.8571 & 0.0534 \\
\hline $\mathrm{a} 3$ & 2.5197 & 2.3800 & 4.8997 & 0.1397 \\
\hline $\mathrm{b} 1$ & 2.4207 & 2.3739 & 4.7946 & 0.0468 \\
\hline $\mathrm{b} 2$ & 2.4069 & 2.1974 & 4.6044 & 0.2095 \\
\hline $\mathrm{b} 3$ & 2.1444 & 1.9709 & 4.1153 & 0.1735 \\
\hline $\mathrm{b} 4$ & 2.0873 & 2.1650 & 4.2523 & -0.0777 \\
\hline $\mathrm{c} 1$ & 2.1148 & 2.5115 & 4.6264 & -0.3967 \\
\hline $\mathrm{c} 2$ & 2.1168 & 2.0056 & 4.1225 & 0.1112 \\
\hline $\mathrm{d} 1$ & 2.1204 & 2.2455 & 4.3659 & -0.1251 \\
\hline $\mathrm{d} 2$ & 2.1690 & 2.1550 & 4.3240 & 0.0140 \\
\hline
\end{tabular}

Calculate Table IV and sort out the sums of rows and lines to obtain Table V.

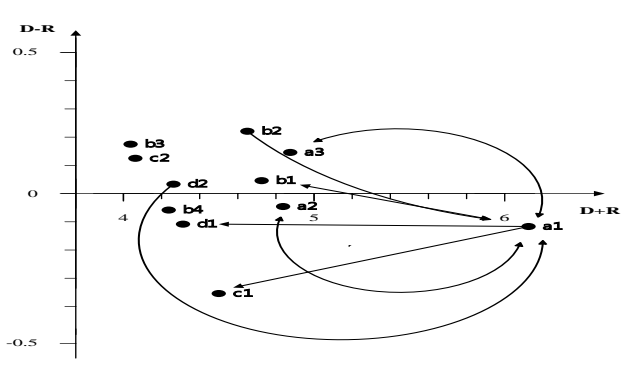

Figure 3. Causal Diagram of Criteria

The relational matrix of overall influence of the adolescent Internet counseling strategy is obtained by summarizing the above listed items and the threshold is calculated as 0.2875 by means of MMDE. And then, the values of $D$ and $R$, as well as the prominence $(D+R)$ and relation (D-R) are calculated. The greater the $D+R$ value is, the greater the importance of the criterion in the overall assessment is. The first three items in the 11 criteria are [a1 regulation and restraint], [a3 proper environment planning] and [a2 learning effective methods to communicate with adolescents] in this order. In addition, as for the value (D-R) obtained by 
subtracting the sum of rows from that of lines, the greater the positive value of $\mathrm{D}-\mathrm{R}$ is, the easier it is for the criteria to directly influence other factors; the greater the negative value of $D-R$ is, the easier it is for the criteria to be directly influenced by other factors. The first three items of the positive values of $D-R$ are $[\mathrm{b} 2$ providing the adolescents with selective and substitute activities], [b3 strengthening the adolescents' establishment of interpersonal relationships and training their skills to communicate] and [a3 proper environmental planning] in this order. The first three items of the negative values of D-R are respectively [c1 creating a good network environment], [a1 regulation and restraint] and [d1 strengthening the mechanism of Internet crime]. Moreover, when $\mathrm{D}+\mathrm{R}$ and $\mathrm{D}-\mathrm{R}$ are combined, only [a3 proper environment planning] is discovered as consistent among the critical criteria of which the degrees of relevance are higher and the degrees of influence are positive and higher. Besides, the criterion that the first three items of the ranking of $D+R$ once again explains that the dimension of [family] plays a crucial role in adolescent Internet addiction.

\section{CONCLUSION AND SUGGESTION}

In this study, the dimension [family] is found as the most important factor in adolescent Internet addiction counseling through the analysis of DEMATEL. The research of Chang [22] indicates that the adolescent Internet addiction tends to be closely related to family factors including the atmosphere and cohesion in the family, the care and communication between parentchild interaction and so on, all of which tend to be significantly related to addiction, mutually supported by the study results. In terms of prominence of $D+R$, the criteria [a1 regulation and restraint], [a3 proper environment planning] and [a2 learning effective methods to communicate with adolescents] under the dimension [family] are indeed the most important and will have positive influence on [school] and [environment]. The study shows that the weight of D+R of the criterion [a1 regulation and restraint] is 6.37, which means it is necessary to properly control and restrain the adolescent's use of the Internet; tutors or parents may place the computers where the parents can control them easily, such as study rooms or living rooms, which can not only control the time that the adolescent spend online but also properly monitor or participate in what the children are surfing for. Family is a significant lin $\mathrm{k}$ to enabling the adolescent to have the correct notion and action with regard to Internet use. What the school is able to do is offering the youth with some selective and substitute activities, such as community activities, summer camps, etc.; however, the adolescent would not care about other activities if they are immersed online, for this reason, the family plays a basic and important role in the adolescent Internet addiction counseling. As restrained by human and material resources, the study can only take the scale as self-reported by the researchers or counselors related to Internet addiction according to their o wn practical experiences as the study tool, so the deduction is restricted; the subsequent researchers have suggested to make the discussion from other aspects. For example, a more complete index of Internet addiction counseling strategy is obtained by discussing from the perspectives of parents and the consciousness, understanding, maturity, etc. of the adolescents themselves on Internet addiction, which is more conductive to the empirical result.

\section{REFERENCES}

[1] TWNIC, "The Research of Taiwan Internet Use of the environment and behavior," Taiwan Network Information Center, 2010.

[2] J. H. Wang, "The study of use internet consultation for youngsters with internet behavior," Paper presented at the Youth issues related to Internet use and countermeasures Seminar, 2004, from http://www.heart.net.tw/wang/paper- new/paper 20040402.htm.

[3] A. Joinson, Causes and implications of disinhibited behavior on the Internet. In J. Gackenbach, et al. (Eds.), Psychology and the Internet: Intrapersonal,interpersonal, and transpersonal implications, 1998, 43-60, San Diego: Academic Press, Inc.

[4] K. S. Young, Internet can be as addicting as alcohol, drugs and gambling, says new research, 1996, from http://www.apa.org. Directory:rele ases File:internet. html. 3972.

[5] J. I. Shieh, H. H. Wu and K. K. Huang, “A DEMATEL method in identifying key success factors of hospital service quality," KnowledgeBased Systems, 2010, 23(3), 277-282.

[6] M. L. Tseng, and Y. H. Lin, "Application of fuzzy DEMATEL to develop a cause and effect model of municipalsolid waste management in Metro Manila," Environmental Monitoring and Assessment, 2009, 158(1-4), 519-533.

[7] R. Fekri, A. A liahmadi and M. Fathian, "Identifying the cause and effect factors of agile NPD process with fuzzy DEMATEL method: the case of Iranian companies," Journal of Intelligent Manufacturing, 2009, 20(6), 637-648.

[8] C.A. Chen, Super DEMATEL v1.6 Program, 2012, from mail:cachen@chu.edu.tw.

[9] K.S. Young, Caught in the net: How to Recognize the Signs of Internet Addiction, New York: John Wiley, 1998.

[10] Young, K. S., "Internet addiction: Evaluation and treatment," Student British Medical Journal, 1999, 7, 351-393.

[11] J. M. Grohol, Internet Addiction Guide, 2005, Retrieved August 30, 2008, from http://psychcentral.com/netaddiction/.

[12] H.I. Wei, J. J. Li, and W. W. Wang, "Risk Factors and Delinquent Behavior Associated with Internet Addiction among High School Students: A Pilot 
Study in Taichung,” Journal of National Taichung

University: Education, 2006, 20(1), 101-102.

[13] C. Zastrow and K. K. Kirst-Ashman, Understanding Human Behavior and the Social Environment, 7e, Cengage Learning, 2009.

[14] J. H. Wang, "Research for caused of Internet addiction and counseling strategy," Guidance Quarterly, 2008, 44(1), 1-12.

[15] A. Gabus, and E. Fontela, "World problems, an invitation to further thought within the framework of DEMATEL," Geneva Research Centre, 1972, 2(1), Switzerland Geneva: Battelle, 4-6.

[16] A. Gabus and E. Fontela, "Perceptions of the world problematique: Communication procedure, communicating with those bearing Collective responsibility," Geneva Research Centre, 1973, 3(2), Switzerland Geneva: Battelle, 10-12.

[17] K. H. Shih, and Y. T. Liu, "A Research on the Competency of Financial Professions," Business Review, 2008, 13(2), 58-60.

[18] T. Y. Li, “Entropy,” MATHMEDIA, 2002, 13(3).

[19] C. W. Li and G. H. Tzeng, "Identification of a threshold value for the DEMATEL method using the maximum mean de-entropy algorithm tfind critical services provided by a semiconductor intellectual property mall," Expert Systems with Applications, 2009, 36, 9891-9898.

[20] M. H. Lee, Nation Branding of Taiwan from Tourism, Chung Hua University, Hsin Chu, Taiwan, 2010.

[21] J. Y. Teng, Project evaluation: methods and applications, National Taiwan Ocean University, Keelung, Taiwan, 2002.

[22] K. P. Chang, “A Study on Parental Rearing Attitude, Family Mental Environment, and Teenager's Internet Addiction," Journal of Family Education and Counseling, 2009, 6, 111.

Dr. Chun-An Chen is an Associate Professor in Chung Hua University, Taiwan. He received his BS degree in Mechanical Engineering and MA, PhD in Management from National Chiao Tung University in Taiwan, and has been working in the Industrial Technology Research Institute. His research interest focuses on tourism strategy management. And he has published some papers about tourism issue on SSCI journals. His research interest focuses on DEMATEL, MMDE, AHP and ANP approaches.

Dr. Hsien-Li Lee is an Assistant Professor in Chung Yuan Christian University, Taiwan. She received her PhD in Accounting from National Taipei University and MA in Management from National Chiao Tung University.

Mr. Wen-Bin Yuan has MBA degree from Chung Hua University, Taiwan. 